\title{
LA CRISIS DEL COVID-19 Y SUS SECUELAS EN LA IGUALDAD DE GÉNERO \\ Un análisis de la mujer en la región del Norte de África y Medio Oriente
}

\author{
THE COVID-19 CRISIS AND ITS SEQUELS IN GENDER EQUALITY \\ An analysis of women in the North Africa and Middle East region
}

\author{
Lidia Guardiola Alonso*
}

\begin{abstract}
RESUMEN: El impacto de la pandemia provocada por el COVID-19 ha llevado al mundo a una crisis sanitaria y de emergencia sin precedentes. En este escenario, las mujeres de la región del Norte de África y Medio Oriente han experimentado una acentuación de la ya existente brecha de género. El aumento de las desigualdades se ha podido observar en múltiples ámbitos como el económico, el sanitario o el social. Asimismo, se ha producido una exacerbación de problemáticas como la violencia de género, ya conocida como "la pandemia en la sombra". Pese a las medidas impuestas por los gobiernos y las sugeridas por las distintas Organizaciones Internacionales, la región del Norte de África y Medio Oriente aún debe avanzar en la protección de los derechos humanos de las mujeres.
\end{abstract}

ABSTRACT: The impact of the COVID-19 pandemic has led the world to an unprecedented health and emergency crisis. In this scenario, women in the North African and Middle East region have experienced an accentuation of the already existing gender gap. Rising inequalities have been observed in multiple areas such as economic, health or social. There has also been an exacerbation of issues such as gender-based violence, already known as "the shadow pandemic". Despite the measures imposed by governments and those suggested by different International Organizations, the North African and Middle East region still needs to make progress in protecting women's human rights.

PALABRAS CLAVE: Mujeres, igualdad de género, Norte de África, Medio Oriente, COVID-19, pandemia, derechos humanos.

KEYWORDS: Women, gender equality, North Africa, Middle East, COVID-19, pandemic, human rights.

Fecha de recepción: 15/02/2021

Fecha de aceptación: 15/02/2021

doi: https://doi.org/10.20318/universitas.2021.6201

*Estudiante del Programa de Doctorado en Estudios Avanzados en Derechos
Humanos, Universidad Carlos III de Madrid. E-mail: Iguardiolaalonso@gmail.com 


\section{1.- INTRODUCCIÓN}

El estallido de la pandemia del COVID-19 en diciembre de 2019 en Wuhan (China) ha llevado a toda la sociedad en su conjunto a una situación de emergencia y crisis sanitaria. En medio de este escenario, las mujeres se han visto y siguen viendo especialmente afectadas debido al impacto exacerbado y profundización de las desigualdades entre géneros.

En este sentido, la ONU se ha pronunciado desde abril de 2020 en multitud de ocasiones con la intención de poner el foco en la necesidad de prestar una mayor atención a la igualdad y los derechos de las mujeres para evitar su retroceso a causa de la pandemia. Un informe realizado por la organización en el marco de los ODS $^{1}$ ha instado a las autoridades responsables en la toma de decisiones a hacer hincapié en tres temas transversales ${ }^{2}$ :

1) Inclusión de las mujeres en los procesos de toma de decisiones en la respuesta al COVID-19

2) Transformación de las desigualdades de género emanadas del trabajo de cuidados no remunerado

3) Planteamiento de los paquetes socioeconómicos de abordaje de las consecuencias derivadas del COVID-19 con un enfoque de género, atendiendo tanto a mujeres como a niñas

Este impacto desproporcionado hacia las mujeres no es nada novedoso y puede ser explicado a través del planteamiento dado por JULIA SMITH, que un año antes de la expansión del COVID-19 por todo el mundo ya escribía sobre la "tiranía de lo urgente"3. Este concepto ilustraba la política adoptada por los gobiernos a la hora de abordar necesidades biomédicas en brotes de enfermedades como el Ébola (2014-2016) o el Zika (2015-2016), en detrimento de las cuestiones estructurales como el caso del género, escudándose en la urgencia de dar respuestas sanitarias ${ }^{4}$. Este fenómeno se expandía también a las secuelas posteriores, donde la creación de documentos y estudios que se refirieran al análisis de género de manera desagregada eran prácticamente nulos, reinando el silencio y la

1 En concreto el Objetivo número 5, encargado de lograr la igualdad entre los géneros y empoderar a todas las mujeres y las niñas.

2 ONU, 'Policy Brief: The Impact of COVID-19 on Women' (ONU, 2020) <https://www.un.org/sites/un2.un.org/files/policy_brief_on_covid_impact_on_wom en_9_april_2020.pdf> acceso el 1 de febrero de 2021, p. 3.

3 Julia Smith, 'Overcoming The 'Tyranny Of The Urgent': Integrating Gender Into Disease Outbreak Preparedness And Response' (2019) 27 Gender \& Development, p. 357.

4 Ídem. 
omisión deliberada del análisis de género en el brote de enfermedades ${ }^{5}$.

Así, la pandemia producida por el COVID-19 se instalaría como una crisis dentro de otra ya preexistente conformada por la desigualdad de la mujer, produciendo una acentuación en la brecha de género.

En este sentido, el presente artículo tratará de desengranar cada una de las particularidades de las mujeres en la región específica del Norte de África y Medio Oriente a raíz de la expansión del COVID-19, así como las medidas ya impuestas por gobiernos y organizaciones, para tratar de sintetizar los puntos clave y dilucidar el posible escenario futuro en base a los datos proporcionados en la actualidad.

\section{2.- LA SITUACIÓN DE LAS MUJERES EN EL NORTE DE ÁFRICA Y MEDIO ORIENTE ANTES DE LA PANDEMIA DEL COVID-19}

Las mujeres en la región del Norte de África y Medio Oriente (también apodada como región $\mathrm{MENA}^{6}$ ) se han encontrado tradicionalmente en una situación de especial vulnerabilidad. Pese a que en los últimos años se habían realizado pequeños avances por los derechos de las mujeres (en su mayor parte gracias a los movimientos y organizaciones de mujeres existentes ${ }^{7}$ ), éstos se vieron paralizados debido a múltiples factores como: a) la constante represión hacia las activistas en numerosos países, como Arabia Saudí o Irán, b) la irregular e insuficiente puesta en práctica de los avances y reformas, c) la discriminación de género tanto en la ley como en la práctica, fundamentalmente en lo referente a la herencia, la custodia, el matrimonio y el divorcio y, d) la pasividad y ausencia de herramientas para combatir la violencia de género y sexual ${ }^{8}$.

Como ya se ha hecho alusión, la sociedad ya se había enfrentado con anterioridad a otros brotes de enfermedades pandémicas como el Ébola o el Zika, donde el primero tuvo una presencia innegable en esta región específica. Durante el periodo en el que se desarrolló la enfermedad, en el territorio se produjo el cierre de los centros educativos, lo que conllevó en las niñas un mayor riesgo de verse sometidas al matrimonio infantil, los

\footnotetext{
5 Ídem.

6 La región MENA, alude a los países de Medio Oriente y el Norte de África, específicamente a Arabia Saudí, Argelia, Bahrein, Djibouti, Egipto, Irán, Iraq, Jordania, Kuwait, Líbano, Libia, Mauritania, Marruecos, Omán, Palestina, Qatar, Siria, Túnez, Emiratos Arabes Unidos y Yemen. En este artículo también se incluirá al Sáhara Occidental como parte de la región pese a que los documentos oficiales no lo hagan por su calificación como Territorio No Autónomo.

7 Amnistía Internacional, 'Los Derechos Humanos En Oriente Medio Y El Norte De África' (Amnistía Internacional, 2020)

<https://www.amnesty.org/download/Documents/MDE0113572020SPANISH.pdf> acceso el 1 de febrero de 2021, p. 6.

8 Ídem.
} 
embarazos, la explotación sexual, la mutilación genital femenina o las enfermedades de transmisión sexual, como ponen de manifiesto CRISTINA DÍAZ y NICOLETTA GALANZINI ${ }^{9}$. Además, elevó el riesgo de que esas niñas no pudieran regresar a las escuelas posteriormente, al tener las responsabilidades de los trabajos de cuidados ${ }^{10}$.

Igualmente, la falta de protección a las mujeres y a las niñas desde el principio de la enfermedad del Ébola se hizo evidente al atender los datos extraídos posteriormente ${ }^{11}$, los cuales evidenciaron un incremento en la violencia de género ${ }^{12}$ y una exposición mayor de las mujeres por su posición en primera línea sanitaria, en los trabajos de cuidados o en la mortalidad de madres y neonatos ${ }^{13}$.

Paralelamente, no podemos olvidar el impacto económico que las desigualdades de género han tenido históricamente en las mujeres de la región del Norte de África y Medio Oriente.

\begin{tabular}{l|lllll} 
MEDIO ORIENTEY & 1995 & 2000 & 2005 & 2010 & 2014 \\
NORTE DE ÁFRICA & $(\$ 2014)$ & $(\$ 2014)$ & $(\$ 2014)$ & $(\$ 2014)$ & $(\$ 2014)$ \\
\hline $\begin{array}{l}\text { Pérdida de capital } \\
\text { humano (billones } \\
\text { de dólares) }\end{array}$ & 1.6 & 2.1 & 2.4 & 2.7 & 3.1 \\
$\begin{array}{l}\text { Pérdida de capital } \\
\text { humano per } \\
\text { cápita (\$) } \\
\% \text { de pérdida de } \\
\text { riqueza total }\end{array}$ & 9,275 & 11,261 & 11,220 & 11,150 & 11,757 \\
\end{tabular}

Fuente: Banco Mundial, 2018.

Fruto de las desigualdades entre géneros en lo que respecta a los salarios, no solo las mujeres se han visto afectadas por sus bajos

\footnotetext{
9 Cristina Díaz y Nicoletta Galanzini, 'Una Pandemia En La Sombra De La COVID-10' [2020] Fundación Mujeres por África, p. 6.

10 OCDE, 'COVID-19 Crisis In The MENA Region: Impact On Gender Equality And Policy Responses' (Organización para la Cooperación y el Desarrollo Económicos (OCDE, 2020) <https://read.oecd-ilibrary.org/view/?ref=134_134470w95kmv8khl\&title=COVID-19-crisis-in-the-MENA-region-impact-on-genderequality-and-policy-responses> acceso el 1 de febrero de 2021, p. 4.

${ }_{11}$ Al hacer referencia a los datos, debe aclararse que en el proceso de recogida y elaboración de los mismos no se tuvo en cuenta en ningún momento la necesidad de desagregar por género, lo cual dificultó enormemente el entendimiento total de la situación de las mujeres y las niñas durante la pandemia del Ébola. En IASC, 'Humanitarian Crisis In West Africa (Ebola). Gender Alert' (Inter-Agency Standing Committee (IASC, 2014) <https://www.globalhealth.org/wp-content/uploads/IASCGender-Reference-Group-Gender-Alert-WEST-AFRICA-EBOLA-19-Sept-2014.pdf> acceso el 1 de febrero de 2021, p. 1.

12 Cristina Díaz y Nicoletta Galanzini, 'Una Pandemia En La Sombra De La COVID$10^{\prime}$, cit, p. 6.

13 Sheila Fernández-Luis y otras, '¿Qué Sabemos Del Impacto De Género En La Pandemia De La COVID-19?' [2020] ISGlobal, Serie Covid-19 y estrategia de respuesta, p. 1.
} 
ingresos, sino que los propios países de la región han visto cómo sus economías han perdido un promedio de 3.1 billones de dólares al año en términos de capital humano, según las cifras aportadas por un informe del Banco Mundial del año 2018 ${ }^{14}$. Asimismo, la estimación de la pérdida de riqueza total del último año estudiado databa de un total de un $7.4 \%{ }^{15}$.

Como recoge la OCDE, esta serie de problemas se han visto de la misma manera agravados por la existencia de normas sociales y legales restrictivas en muchos de los Estados parte de la región del Norte de África y Medio Oriente, las cuales han provocado una gran divergencia en el posicionamiento de las mujeres en la sociedad, que se ha visto aún más reforzada con la aparición del COVID-1916.

\section{3.- LA SITUACIÓN DE LAS MUJERES EN EL NORTE DE ÁFRICA Y MEDIO ORIENTE A RAÍZ DE LA PANDEMIA DEL COVID-19}

Es indudable que la crisis provocada por el COVID-19 ha sacudido al presente y el futuro de la población mundial. Los números de personas infectadas y fallecidas han ido en aumento conforme han transcurrido los meses, donde la región del Norte de África y Medio Oriente ha experimentado una acuciante expansión a lo largo y ancho de sus países. La llegada de la pandemia a la región ha profundizado su ya frágil situación fruto de numerosos factores como la presencia prácticamente generalizada del conflicto, la migración, los desplazamientos o incluso la escasez de recursos básicos como la comida o el agua ${ }^{17}$.

En este escenario, han sido las mujeres las que han sufrido en mayor medida las consecuencias traídas por la pandemia en ámbitos como la salud, el trabajo o la violencia de género. Los problemas estructurales y el arraigo de los roles de género han llevado a las mujeres de la región a enfrentar unos mayores y peores efectos.

Las mujeres de esta región, como prácticamente alrededor del globo, se han encontrado en primera línea en el plano de la salud para hacer frente al COVID-19, soportando además un incremento y exacerbación en el trabajo no remunerado y en la violencia de

\footnotetext{
14 Wodon Q y de la Brière B, 'Unrealized Potential: The High Cost Of Gender Inequality In Earnings' (Banco Mundial, 2018) <https://openknowledge.worldbank.org/bitstream/handle/10986/29865/126579-

Public-on-5-30-18-WorldBank-GenderInequality-Brief-

v13.pdf?sequence $=1$ \&isAllowed $=y>$ acceso el 1 de febrero de 2021, p. 9.

15 Ídem.

16 OCDE, 'COVID-19 Crisis In The MENA Region: Impact On Gender Equality And Policy Responses' (Organización para la Cooperación y el Desarrollo Económicos (OCDE, 2020), cit., p. 2.

${ }_{17}$ CARE, 'Rapid Gender Analysis - COVID-19. Middle East And North Africa Region' (CARE International 2020) <https://reliefweb.int/sites/reliefweb.int/files/resources/CARE-MENA-COVID-19RGA-200720201.pdf> acceso el 1 de febrero de 2021, p. 4.
} 
género, como ha puesto de manifiesto el informe elaborado por la OCDE en junio de $2020^{18}$.

Estos factores han situado a las mujeres en una situación de especial vulnerabilidad, pues son las que han mostrado una sobrerrepresentación tradicional en aquellos sectores que han incluido la inseguridad laboral, de ingresos, trabajos domésticos o incluso el contexto de personas refugiadas ${ }^{19}$.

De este modo, las próximas páginas se centrarán en los focos primordiales que han afectado a las mujeres de la región del Norte de África y Medio Oriente en el desarrollo de la pandemia provocada por el COVID-19.

\section{1.- La sobreexposición y vulnerabilidad de las mujeres al COVID-19 a causa de los trabajos feminizados} 3.1.1.- Las mujeres en primera línea de respuesta en la crisis
sanitaria

En el transcurso de los meses durante los que se ha desarrollado el COVID-19, se ha puesto de manifiesto la figura de las mujeres como las principales protagonistas en la primera línea de respuesta en el ámbito sanitario no solo en la región del Norte de África y Medio Oriente, sino en la mayor parte del mundo. Esto las ha llevado a encontrarse en una posición de mayor riesgo a infectarse por la enfermedad del COVID-1920.

Estos hechos han contrastado con los habitualmente bajos salarios percibidos por las mujeres en este sector, que además de encontrarse por debajo de lo común, también han sido invisibilizados y no tenidos en cuenta 21 , como gran parte de los trabajos feminizados. Con la llegada del COVID-19, esta discriminación en el sueldo ha amenazado con acentuarse aún más, pudiendo superar el

18 OCDE, 'COVID-19 Crisis In The MENA Region: Impact On Gender Equality And Policy Responses' (Organización para la Cooperación y el Desarrollo Económicos (OCDE), 2020), cit., p. 2.

19 OCDE, 'COVID-19 crisis response in MENA countries' (Organización para la Cooperación y el Desarrollo Económicos (OCDE), 2020) <https://read.oecdilibrary.org/view/?ref=129_129919-4li7bq8asv\&title=COVID-19-Crisis-Response-inMENA-Countries $>$ acceso el 1 de febrero de 2021

${ }^{20}$ Carolina Rivera y otras, 'Gender Inequality And The COVID-19 Crisis: A Human Development Perspective' (Programa de las Naciones Unidas para el Desarrollo (PNUD), 2020) <http://hdr.undp.org/sites/default/files/covid-

19_and_human_development_-_gender_dashboards_final.pdf $>$ acceso el 1 de febrero de 2021, pp. 4-5.

${ }^{21}$ Interagency Network on Women and Gender Equality (IANWGE), 'IANWGE Compendium on Integrating Gender Considerations in the Response to COVID-19: Key Messages and Actions from Entities' (Interagency Network on Women and Gender Equality (IANWGE), 2020) <https://www.unwomen.org/-

/media/headquarters/attachments/sections/library/publications/2020/ianwgecompendium-on-integrating-gender-considerations-in-the-response-to-covid-19en.pdf?la=en\&vs=5806 $>$ acceso el 1 de febrero de 2021, p. 73. 
$28 \%$ de brecha salarial actual en la región del Norte de África y Medio Oriente 22 .

Estos datos generales de la región han tenido sus especificidades según el país, aunque todos han seguido el mismo patrón de brecha salarial y sobrerrepresentación femenina en la primera línea de respuesta en la salud.

Un ejemplo de ello ha sido el caso de Marruecos. En el país del Norte de África, y atendiendo a los datos brindados por ONU Mujeres, las trabajadoras representan el $57 \%$ del personal médico y el $66 \%$ del personal paramédico, lo cual las ha dejado en un escenario de indefensión ante el virus debido a la sobrecarga del sistema sanitario, la cual ha disminuido notablemente sus capacidades ${ }^{23}$.

En Líbano, el porcentaje de mujeres dentro del personal médico en primera línea asciende a un $70 \%$. Asimismo, un $80 \%$ de las enfermeras son mujeres, de las cuales un $50 \%$ ha acusado estar recibiendo un salario muy reducido antes de la pandemia ${ }^{24}$.

En Egipto y Palestina, el porcentaje de mujeres en el sector de primera línea sanitaria se encuentra situado en un $70 \%{ }^{25}$ y $60 \%{ }^{26}$, respectivamente. La principal problemática en ambos países es la alta participación de los hombres en los niveles más altos de decisión ${ }^{27}$ (incluida la sanitaria), mientras que las mujeres enfrentan unos niveles más bajos pero un mayor riesgo y exposición.

\subsection{2.- El trabajo de cuidados y doméstico}

Igual que ha sucedido con el sector sanitario en primera línea, los trabajos de cuidados y domésticos, usualmente no remunerados, han

22 OCDE, 'COVID-19 Crisis In The MENA Region: Impact On Gender Equality And Policy Responses' (Organización para la Cooperación y el Desarrollo Económicos (OCDE), 2020), cit., p. 12.

23 ONU Mujeres, 'COVID-19 Crisis Update And UN Women Response Morocco' (ONU Mujeres, 2020) <https://www2.unwomen.org/-

/media/field\%20office\%20arab\%20states/attachments/publications/2020/04/covid $19 \% 20$ crisis\%20update\%20and\%20un\%20women\%20response160420.pdf?la=en \&vs $=5039>$ acceso el 1 de febrero de 2021, p. 1 .

${ }^{24}$ ONU Mujeres, 'Women's needs and gender equality in Lebanon's COVID-19 response' (ONU Mujeres, 2020) <https://www2.unwomen.org/-

/media/field\%20office\%20arab\%20states/attachments/publications/2020/03/updat ed\%20lebanon\%20brief/gender\%20and\%20covid_english.pdf?la=en\&vs=403> acceso el 1 de febrero de 2021, p. 4.

25 ONU Mujeres, 'Women's needs and gender equality in Egypt's COVID-19 response' (ONU Mujeres, 2020) <https://www2.unwomen.org/-

/media/field\%20office\%20arab\%20states/attachments/publications/2020/04/unwo men_brief_covid-19_egypt_response.pdf?la=en\&vs=3919> acceso el 1 de febrero de 2021 , p. 2

26 ONU Mujeres, 'COVID-19: Gendered Impacts of the Pandemic in Palestine and Implications for Policy and Programming Findings of a Rapid Gender Analysis of COVID-19 in Palestine' (ONU Mujeres, 2020) <https://www.un.org/unispal/wpcontent/uploads/2020/06/UNWOMENRPT_090620.pdf> acceso el 1 de febrero de 2021, p. 16.

27 Ídem. 
sido trabajos considerados como feminizados. Estas ocupaciones han generado una desigualdad y vulnerabilidad de base en el acceso a oportunidades laborales y al mercado de trabajo 28 , ya que tradicionalmente se ha relegado a la mujer de la región a un tipo de trabajo más precario e invisibilizado, suponiendo la pandemia actual un gran y desproporcionado choque a su presente y futuro.

De hecho, la región del Norte de África y Medio Oriente ostenta la segunda mayor brecha de género en el mundo en lo que respecta a los trabajos de cuidados y domésticos no remunerados ${ }^{29}$, solo superada por la región del Sudeste Asiático ${ }^{30}$.

En el marco del COVID-19, las mujeres han afrontado una mayor carga en los cuidados con el confinamiento, pues las tareas han aumentado y se han intensificado, en muchas ocasiones por los cierres de los centros educativos y la obligación de las y los menores de quedarse en sus hogares ${ }^{31}$.

Asimismo, este tipo de trabajos han presentado un especial riesgo para las mujeres refugiadas de la región, las cuales han estado ligadas a los mismos, principalmente al sector agricultor y el doméstico. La precarización emanada de la crisis sanitaria provocada por el COVID-19 ha impactado con mayor fuerza a las mujeres refugiadas, pues ya partían de una situación anterior mucho más delicada e inestable ${ }^{32}$.

Paralelamente, el confinamiento ha llevado a un proceso de aprendizaje y trabajo on-line por la incapacidad de salir debido a las restricciones de movimiento. En este escenario, las mujeres también han sufrido un impacto desproporcional, ya que en los hogares en los que se ha podido tener un acceso a un ordenador las normas sociales han favorecido a los hombres, permitiendo que sean ellos los que accedan a los mismos ${ }^{33}$, mientras que las mujeres y las niñas se han visto usualmente sin acceso a internet ${ }^{34}$.

28 M Solanas Cardín, 'La Crisis Del COVID-19 Y Sus Impactos En La Igualdad De Género' [2020] Real Instituto Elcano, p. 2.

29 Las mujeres de la región del Norte de África y Medio Oriente emplean alrededor de 5 horas diarias en los trabajos no remunerados, mientras que los hombres se posicionan en un poco más de una hora diaria. En OCDE, 'COVID-19 Crisis In The MENA Region: Impact On Gender Equality And Policy Responses' (Organización para la Cooperación y el Desarrollo Económicos (OCDE), 2020), cit., p. 13.

30 Ídem.

31 Interagency Network on Women and Gender Equality (IANWGE), 'IANWGE Compendium On Integrating Gender Considerations In The Response To COVID-19:

Key Messages And Actions From Entities' (Interagency Network on Women and Gender Equality (IANWGE), 2020), cit., p. 54.

32 OCDE, 'COVID-19 Crisis In The MENA Region: Impact On Gender Equality And Policy Responses' (Organización para la Cooperación y el Desarrollo Económicos (OCDE), 2020), cit., p. 8.

${ }^{33}$ Como pone de ejemplo un informe creado por ONU Mujeres, en el caso de Iraq un $98.3 \%$ de los hombres tiene acceso a internet, frente al $51.2 \%$ de las mujeres.

En ONU Mujeres, 'The impact of COVID-19 on Gender Equality in the Arab Region' (ONU Mujeres, 2020) <https://www2.unwomen.org/-

/media/field\%20office\%20arab\%20states/attachments/publications/2020/04/impac 
Esta coyuntura anterior ha sucedido en los casos en los que el núcleo familiar ha podido acceder a un ordenador. No obstante, también se ha dado la circunstancia contraria en la cual las mujeres, debido a su situación de pobreza por su posición como trabajadoras con una escasa o incluso nula remuneración (además de su discriminación de base), no han podido costearse un ordenador ni ningún medio para poder trabajar o estudiar desde el confinamiento en sus hogares ${ }^{35}$.

Ambas tesituras son de especial riesgo no solo para el ámbito laboral y educativo de las mujeres y las niñas en el periodo que dure la pandemia del COVID-19, sino también para la fase posterior al mismo, donde presumiblemente tendrán una gran dificultad para poder retomar los estudios o encontrar un trabajo ${ }^{36}$.

Mientras que la asimetría laboral y desigualdad de género han ido en aumento a causa de las secuelas provocadas por el COVID-19, las cargas producidas por el trabajo de cuidados y doméstico no remunerado han dejado con más responsabilidades y en una mayor vulnerabilidad a las mujeres en la región del Norte de África y Medio Oriente.

\section{2.- Las secuelas para las mujeres y las niñas en el ámbito educativo}

Con el decreto de medidas para intentar mitigar los efectos del COVID-19, los centros educativos se han visto obligados a cerrar y dejar de dar clases a los niños y las niñas en gran parte de los países del mundo, trasladando sus aulas al espacio on-line.

En el caso de la región del Norte de África y Medio Oriente, y como se ha mencionado en el apartado anterior, la posibilidad de tener un ordenador y conexión a internet es baja y en el caso de tenerlos, las normas sociales discriminatorias llevan a que sean los hombres los que tengan acceso a ellos en la mayoría de los casos.

Además, las niñas también soportan una mayor carga en los trabajos domésticos y de cuidados, llevando a un menor tiempo y energía para poder dedicarlo en su educación desde sus casas ${ }^{37}$.

Volviendo de nuevo a las cifras aportadas por el brote de Ébola, en los dos años de incidencia de la pandemia los embarazos

t\%20of\%20covid\%20on\%20gender\%20equality\%20\%20policy\%20brief.pdf?la=en $\& v s=4414>$ acceso el 1 de febrero de 2021, p. 3.

${ }^{34}$ Ibidem, pp. 3-4.

35 Ídem.

36 Ídem.

37 UNFPA, ' Daring to Ask, Listen, and Act: A Snapshot of the Impacts of COVID-19 on Women and Girls' rights and sexual and reproductive health' (United Nations Population Fund (UNFPA), 2020) <

https://reliefweb.int/sites/reliefweb.int/files/resources/20200511_Daring\%20to\%2 0ask\%20Rapid\%20Assessment\%20Report_FINAL.pdf> acceso el 1 de febrero de 2021 , p. 16. 
adolescentes crecieron en un $65 \%,{ }^{38}$. Un dato que muy probablemente pueda repetirse de nuevo con esta nueva pandemia.

Además, un total de 11.000 embarazos fueron de niñas menores de edad, suponiendo un $79 \%$ del total (tan solo 3.000 embarazos fueron de mujeres mayores de edad en este periodo) ${ }^{39}$. Una de las causas de estas consecuencias se produjo por el cierre de los centros educativos, los cuales llevaron a un escenario de marginación a las niñas de la región.

Por último, un problema que tampoco puede pasar desapercibido es el de la utilización de los centros educativos como medios de denuncia ante situaciones de violación de los derechos de las niñas, como ponen de relieve CRISTINA DÍAZ y NICOLETTA GALANZINI ${ }^{40}$. Al encontrarse el $90 \%$ de las niñas residentes en países africanos confinadas en sus respectivos hogares por las medidas implantadas por el COVID-19, su protección en caso de vulneración de alguno de sus derechos se torna enormemente complicada 41 .

\section{3.- Los derechos reproductivos y sexuales de las mujeres}

La pandemia provocada por el COVID-19 ha azotado a las mujeres de la región en todos los ámbitos posibles, siendo uno de los más delicados el de los derechos reproductivos y sexuales de las mujeres, así como el acceso a la sanidad en general. Dada la crisis sanitaria actual, los principales esfuerzos han ido destinados a tratar de detener la expansión del virus, dando una menor prioridad al acceso a la salud de las mujeres, fundamentalmente los servicios que incluyen sus derechos reproductivos y sexuales ${ }^{42}$. La posición de mayor vulnerabilidad y marginación de las mujeres solteras y las niñas desde antes de la pandemia las ha convertido en un colectivo con mayor riesgo de padecer una exclusión del sistema de salud reproductivo $\mathrm{y} / \mathrm{o}$ sexual ${ }^{43}$.

Según el "Fondo de Población de las Naciones Unidas" en la región, a fecha de 17 de abril de 2020 había un total de 8 millones de mujeres embarazadas y 107 millones de mujeres en edad reproductiva ${ }^{44}$. De todas ellas, más de 15 millones de mujeres en

\footnotetext{
38 Cristina Díaz y Nicoletta Galanzini, 'Una Pandemia En La Sombra De La COVID10 ', cit., p. 6.

39 Ídem.

40 Ibidem, p. 12.

41 Ídem.

42 Rouzeh Eghtessadi y otros, 'Safeguarding Gains In The Sexual And Reproductive Health And AIDS Response Amidst COVID-19: The Role Of African Civil Society' (2020) 100 International Journal of Infectious Diseases, p. 286.

43 ONU Mujeres, 'The impact of COVID-19 on Gender Equality in the Arab Region' (ONU Mujeres, 2020), cit., p. 2.

${ }^{4}$ UNFPA, 'Arab States Region COVID-19. Situation Report No. 1' (United Nations

Population Fund (UNFPA), 2020)
} 
edad reproductiva estaban en necesidad de ayuda humanitaria a causa del COVID-19, de las cuales un millón y medio estaban embarazadas ${ }^{45}$.

La disminución de la financiación de los diferentes países hacia los derechos sexuales y reproductivos de las mujeres y las niñas supondrán previsiblemente un aumento en la mortalidad durante el embarazo y en el parto, en los embarazos de menores y en el contagio de enfermedades de transmisión sexual ${ }^{46}$.

Asimismo, la escasa salud reproductiva y sexual se ha visto agravada en los casos de las mujeres refugiadas, las cuales prácticamente no han tenido acceso ni siquiera a productos básicos de higiene íntima. Las últimas cifras aportadas por los informes de CARE han revelado que en Jordania el $24 \%$ de las refugiadas tienen necesidades de higiene menstrual. De igual manera, las refugiadas sirias en Turquía sin productos de higiene necesario ascienden a un $47 \% 47$.

\section{4.- La violencia de género}

Uno de los objetivos primordiales establecidos por el ODS número 5 (dedicado a la igualdad de género y al empoderamiento de la mujer) en su paquete de medidas para prevenir, mitigar y responder a los efectos del COVID-19 en las mujeres, ha sido precisamente el de reducir la violencia de género. Este especial foco en la violencia de género se ha debido a que desde el inicio de la pandemia ha sido uno de los riesgos que ha afectado a la mayor parte de las mujeres ${ }^{48}$. Tanto es así que ya ha sido apodada como "la pandemia en la sombra" del COVID-1949.

Las situaciones que se han analizado en los anteriores epígrafes referentes a los efectos socioeconómicos en las mujeres muchas veces han derivado en mayores riesgos de sufrir un aumento en la violencia de género. El confinamiento ha provocado que tanto

<https://www.unfpa.org/sites/default/files/resource-pdf/ASRO_COVID-

19_UNFPA_Sitrep_No.1.pdf> acceso el 1 de febrero de 2021, p. 1.

45 Ídem.

46 OCDE, 'COVID-19 Crisis In The MENA Region: Impact On Gender Equality And Policy Responses' (Organización para la Cooperación y el Desarrollo Económicos (OCDE), 2020), cit., p. 15.

47 CARE, 'Rapid Gender Analysis - COVID-19. Middle East And North Africa Region' (CARE International, 2020), cit., p. 18.

48 Los estudios muestras que una de cada tres mujeres han experimentado violencia de género por parte de su pareja íntima alguna vez en su vida. Estos datos aumentan con la aparición de una crisis sanitaria y de emergencia. En Saravana Ravindran y Manisha Shah, 'Unintended Consequences Of Lockdowns: COVID-19 And The Shadow Pandemic' [2020] National Bureau of Economic Research Working Paper Series, p. 2.

${ }^{49}$ Ibidem, p. 16. 
mujeres como niñas queden atrapadas con sus agresores y sin posibilidad de pedir ayuda o escapar ${ }^{50}$.

Por consiguiente, la violencia de género emanada del COVID-19 se ha explicado mediante el supuesto de confinamiento, que al unirse a otros factores como la inseguridad del trabajo, el aislamiento en un hogar cerrado y usualmente pequeño, la imposibilidad de acceder a los servicios de salud, los limitadas vías de atención, el cierre de las escuelas e incluso el deterioro de la salud mental a causa el encierro, han hecho de un caldo de cultivo idóneo para que se sucedan este tipo de violencias. De alguna forma, quienes la cometen buscan su justificación en los anteriores aspectos ${ }^{51}$.

En concreto, AMBER PETERMAN y otras han identificado un total de nueve vías que, de manera directa o indirecta, actúan de vínculo entre la pandemia y la violencia de género, exacerbando los efectos en la segunda. Éstas son: 1) la inseguridad económica y el estrés relacionado con la pobreza, 2) la cuarentena y el aislamiento social, 3) el malestar y la inestabilidad relacionados con los desastres y los conflictos, 4) la exposición a relaciones de explotación debido a los cambios demográficos, 5) el acceso reducido a los servicios de salud y disponibilidad del personal de primera respuesta, 6) la incapacidad de las mujeres para escapar de sus parejas abusivas, 7) las fuentes de violencia específicas del virus, 8) la exposición a la violencia y coerción en los esfuerzos de respuesta, y 9) la violencia perpetrada contra los trabajadores de la salud ${ }^{52}$.

No obstante, el contexto de violencia de género durante la pandemia, así como su alcance y características, son aún desconocidos. Varios informes han recogido que las mujeres en la región podrían estar obligadas por sus agresores a cortar interacciones sociales con su familia incluso por teléfono ${ }^{53}$. No solo eso, sino que los agresores podrían haber cortado la fuente de información a las mujeres para negarles el acceso al conocimiento de la realidad actual o las nuevas posibilidades de denuncia de agresores ${ }^{54}$.

Aunque varios países han tratado de mitigar la situación mediante la creación de servicios de línea directa para recolectar información sobre violencia de género durante el confinamiento y

50 Timothy P. Williams y Kirsten Pontalti, 'Responding To The Shadow Pandemic: Taking Stock Of Gender-Based Violence Risks And Responses During COVID-19. Child Protection Learning Brief' (UNICEF, 2021)

<https://www.unicef.org/media/76916/file/Gender-Based-Violence-in-

Emergencies-CP-Learning-Brief-Aug-2020.pdf> acceso el 1 de febrero de 2021.

51 OCDE, 'COVID-19 Crisis In The MENA Region: Impact On Gender Equality And Policy Responses' (Organización para la Cooperación y el Desarrollo Económicos (OCDE), 2020), cit., p. 14.

52 Amber Peterman y otras, 'Pandemics And Violence Against Women And Children' [2020] Center for Global Development. Working Paper 528, p. 5.

53 ONU Mujeres, 'The impact of COVID-19 on Gender Equality in the Arab Region' (ONU Mujeres, 2020), cit., p. 4.

54 Ídem. 
brindar asistencia, estos han sido insuficientes para frenar el incremento de la violencia en los hogares ${ }^{55}$.

En el caso de Palestina, una organización de mujeres ha instalado una línea directa de ayuda para poder recibir llamadas de mujeres que estén sufriendo violencia de género en el confinamiento. Cuando la línea ha ampliado sus horarios, las llamadas han aumentado un $38 \%$, pudiendo estar la razón en una limitación del acceso al teléfono por parte de los agresores o de privacidad de las mujeres víctimas de violencia de género ${ }^{56}$. Igualmente se han recibido llamadas por casos de intentos de suicido, incesto 0 violaciones ${ }^{57}$.

Ello ha aumentado la vulnerabilidad de las mujeres en la región, en especial la de aquellas en zonas de conflicto armado, refugiadas, trabajadoras domésticas o con discapacidad ${ }^{58}$, las cuales se han visto sobreexpuestas a la explotación sexual debido a su situación de especial vulnerabilidad ${ }^{59}$.

Por último, la violencia de género en la región ha sido en algunas ocasiones degradada a un segundo plano y restada de importancia por la policía y los sistemas de justicia. La no priorización de esta importante violación de los derechos de las mujeres se ha explicado por la no penalización de la violencia de género en muchos países, desamparando a las mujeres en estos escenarios de pandemia ${ }^{60}$.

\section{5.- Las mujeres en zonas de conflicto}

Además de los principales focos de violación de los derechos de las mujeres y las niñas que se han visto exacerbados con la pandemia, no se puede ignorar una constante en esta región y es la del conflicto y la inestabilidad. Pese al estado de emergencia y paralización global, el conflicto no se ha detenido ni siquiera con las

55 CARE, 'Rapid Gender Analysis - COVID-19. Middle East And North Africa Region' (CARE International, 2020), cit., p. 20.

56 ONU Mujeres, 'COVID-19: Gendered Impacts of the Pandemic in Palestine and Implications for Policy and Programming Findings of a Rapid Gender Analysis of COVID-19 in Palestine' (ONU Mujeres, 2020), cit., p. 19.

57 Ídem.

58 Las mujeres con discapacidades intelectuales y cognitivas tienen de dos a cuatro veces más de posibilidades de enfrentar violencia de género en los supuestos del confinamiento. En Cristina Díaz y Nicoletta Galanzini, 'Una Pandemia En La Sombra De La COVID-10', cit. , pp. 1-2.

59 OCDE, 'COVID-19 Crisis In The MENA Region: Impact On Gender Equality And Policy Responses' (Organización para la Cooperación y el Desarrollo Económicos (OCDE), 2020), cit., p. 15.

60 ONU SIDA, 'New Awareness Campaign On Gender-Based Violence In The Middle East And North Africa' (ONU SIDA, 2020)

<https://www.unaids.org/en/resources/presscentre/featurestories/2020/november/ 20201125_awareness-campaign-gender-based-violence-middle-east-north-africa> acceso el 1 de febrero de 2021. 
peticiones del alto al fuego humanitario para conseguir frenar la expansión de la pandemia.

Así, muchos de estos países de la región del Norte de África y Medio Oriente se han visto envueltos en un conflicto armado en el momento del estallido del COVID-19 (algunos desde hace muchos años atrás), como Libia, Siria, Iraq, Palestina, Yemen ${ }^{61}$ o el Sáhara Occidental ${ }^{62}$.

En un escenario de conflicto, los sistemas de salud no funcionan correctamente 63 , agravando aún más todas las situaciones que se han expuesto anteriormente. Asimismo, las medidas impuestas como el distanciamiento, el confinamiento o las mascarillas no son posibles dada la tesitura.

Entrando en el tema que aquí concierne, las mujeres en esta región se han encontrado históricamente expuestas a un mayor riesgo en términos de sanidad, trabajo, educación y violencia de género ${ }^{64}$. En un contexto como el de conflicto o de emergencia humanitaria, las mujeres se han visto en una situación de especial vulnerabilidad, donde el COVID-19 se ha introducido como un factor que la ha agravado aún más si cabe ${ }^{65}$.

Para revertir la situación de especial vulnerabilidad de la mujer en la región es necesario trasladar el papel de la mujer como víctima de conflicto a protagonista en los procesos de paz, de acuerdo a una correcta implementación de la "Resolución 1325 (2000) sobre Mujeres, Paz y Seguridad del Consejo de Seguridad de la ONU". Este cambio no solo se torna necesario para las mujeres en particular, sino para la sociedad en general. Diversas investigaciones han mostrado cómo la inclusión de las mujeres en los procesos de paz han tenido como consecuencia unos índices más altos de permanencia en el

61 ONU Mujeres, 'The impact of COVID-19 on Gender Equality in the Arab Region' (ONU Mujeres, 2020), cit., p. 5.

62 El Sáhara Occidental y Marruecos se encuentran en guerra desde el 13 de noviembre de 2020 tras el incumplimiento por parte de Marruecos del alto al fuego declarado en 1991. La utilización de fuerzas militares marroquíes en la zona de El Guerguerat contra las personas saharauis, que se encontraban allí manifestándose y realizando un bloqueo del paso fronterizo, ha llevado a esta violación del alto al fuego por parte de Marruecos. A diferencia de los demás conflictos, éste ha sido el único que ha entrado en guerra activa en plena pandemia, pues los demás ya se encontraban en ella anteriormente. En Julien Lafontaine Carboni y Juan Carlos Gimeno Martín, 'Inmóviles, Pero No Quietos. La Sedentarización De Los Saharauis Como Estrategia De Adaptación Y Respuesta A La Supervivencia. Sobre La Posibilidad De Un Nomadismo Inmóvil' [2021] Tabula Rasa, p. 45.

63 Francesca Caruso, 'COVID-19 And Conflict Mediation: Women, Not The Pandemic Can Revive Diplomacy' [2020] Istituto Affari Internazionali, 52, p. 1.

64 OCDE, 'COVID-19 Crisis In The MENA Region: Impact On Gender Equality And Policy Responses' (Organización para la Cooperación y el Desarrollo Económicos (OCDE), 2020), cit., pp. 10-11.

65 Kristen Meagher, Neha S Singh and Preeti Patel, 'The Role Of Gender Inclusive Leadership During The COVID-19 Pandemic To Support Vulnerable Populations In Conflict Settings' (2020) 5 BMJ Global Health, p. 1. 
tiempo, así como de incidencia en la presión a las partes y al reanudamiento de las negociaciones ${ }^{66}$.

No obstante, los avances en la implementación de la "Resolución 1325 del Consejo de Seguridad de la ONU" aún no han tenido los progresos esperados. En el caso de las mujeres yemeníes, pese a que han estado presentes en multitud de acciones para intentar frenar los conflictos, no han visto traducidos sus esfuerzos en una inclusión en las conversaciones de paz ${ }^{67}$. Su presencia en actos a nivel local no son suficientes si son constantemente excluidas de los procesos formales.

Por otro lado, con la llegada del COVID-19 la participación de las mujeres en la resolución de conflictos se ha manifestado principalmente por parte de las organizaciones de mujeres. El ejemplo más claro hasta ahora se ha encontrado en la declaración conjunta firmada por un total de noventa y nueve organizaciones de mujeres de Iraq, Libia, Palestina, Siria y Yemen. En la declaración, estas organizaciones de mujeres se han unido al llamamiento al alto al fuego realizado por el Secretario General de la ONU, Antonio Guterres, el 23 de marzo de $2020^{68}$. Además de suscribir las palabras del Secretario General, han destacado el especial sufrimiento de las mujeres y las niñas a causa del aumento de la discriminación, la violencia (incluida la violencia sexual), la trata, el terrorismo o la esclavitud ${ }^{69}$.

Por ende, la participación de las mujeres en el establecimiento de la paz en los conflictos existentes en la región de una manera más activa es especialmente necesaria en tiempos de pandemia.

\section{4.- LA POSIBLE SITUACIÓN FUTURA DE LAS MUJERES EN EL NORTE DE ÁFRICA Y MEDIO ORIENTE FRUTO DE LAS MEDIDAS IMPUESTAS}

\footnotetext{
66 ONU Mujeres, 'COVID-19 y conflictos: Fomentar la participación sustantiva de las mujeres en los procesos de paz y alto el fuego. Documento de políticas no. 19' (ONU Mujeres, 2020) < https://www.unwomen.org/-

/media/headquarters/attachments/sections/library/publications/2020/policy-briefcovid-19-and-conflict-es.pdf?la=es\&vs=3825> acceso el 1 de febrero de 2021, p. 3.

67 Francesca Caruso, 'COVID-19 And Conflict Mediation: Women, Not The Pandemic Can Revive Diplomacy', cit., p. 4.

68 ONU, 'Policy Note on the United Nations Secretary-General's Call for a Global Ceasefire: Challenges and Opportunities' (Mediation Support Unit, Policy \& Mediation Division 2020)

https://peacemaker.un.org/sites/peacemaker.un.org/files/Policy\%20Note\%20Ceas efire\%20Call\%20Challenges\%200pportunities_0_0.pdf > acceso el 1 de febrero de 2021 , p. 2.

69 ONU Mujeres, 'Press Release: Women'S Organizations In The Arab States Region Join UN Secretary-General António Guterres'S Call For Ceasefire In The Face Of COVID-19' (2020) <https://www.unwomen.org/en/news/stories/2020/5/pressrelease-women-organizations-in-the-arab-states-call-for-ceasefire-in-the-face-ofcovid19> acceso el 1 de febrero de 2021
} 
Así, y dada la tesitura que se ha desarrollado a raíz de la pandemia, los diferentes actores y sujetos han intentado dar respuesta a la situación. Por un lado, las mujeres de la sociedad civil han tenido y siguen teniendo un rol imprescindible en la consecución de sus derechos, siendo representadas por algunas organizaciones o redes de organizaciones, como lo ha recogido otro informe elaborado por CARE70. Este hecho ha sido de vital importancia en la respuesta a la pandemia del COVID-19, pues un liderazgo de las mujeres representadas por las organizaciones podrá llevar a un enfoque de género en la respuesta al mismo ${ }^{71}$. De hecho, algunos gobiernos ya han comenzado a trabajar con estas organizaciones de mujeres en la elaboración de una perspectiva de género en la pandemia ${ }^{72}$.

Por otro lado, ya se ha comenzado a perfilar la respuesta por parte de los gobiernos a la crisis producida por el COVID-19, la cual deberá tener integrado un plan con perspectiva de género destinado a reducir la brecha de igualdad y conseguir disminuir los efectos que la pandemia está teniendo en los derechos de las mujeres y las niñas. En este sentido, la "Oficina del Alto Comisionado de las Naciones Unidas para los Derechos Humanos" (ACNUDH) y la "Unión Africana" (UA) ${ }^{73}$ han llevado a cabo la elaboración de un documento en el que han puesto de relieve los 7 puntos principales o líneas de acción que tienen que tener en cuenta los países a la hora de abordar los derechos de las mujeres africanas en la pandemia ${ }^{74}$, los cuales se dividen en:

1. Medidas económicas. Como ya se ha visto anteriormente, los trabajos feminizados han tenido un impacto desproporcionado para las mujeres de la región, aumentando su situación de vulnerabilidad y pobreza. Por ello, el informe de la ONU y la UA ha propuesto una serie de mecanismos destinados a mitigar los efectos socioeconómicos de género, especialmente en el sector informal y a las mujeres con mayores necesidades ${ }^{75}$.

\footnotetext{
${ }^{70}$ CARE, 'One More Setback For Refugees In MENA -Especially Women And Girls' (CARE International, 2020) <https://reliefweb.int/sites/reliefweb.int/files/resources/CARE_MENA_Rapid_Gende r_Analysis_Summary.pdf> acceso el 1 de febrero de 2021.

${ }^{71}$ ONU Mujeres, 'The impact of COVID-19 on Gender Equality in the Arab Region' (ONU Mujeres, 2020), cit., p. 5.

72 OCDE, 'COVID-19 Crisis In The MENA Region: Impact On Gender Equality And Policy Responses' (Organización para la Cooperación y el Desarrollo Económicos (OCDE), 2020), cit., p. 2.

73 Aunque este informe no incluye a Medio Oriente, el impacto en esta zona está siendo similar, por lo que también se pueden trasladar estas acciones de la misma manera que con el Norte de África.

74 ACNUDH y UA, '7 Possible Actions- Women'S Rights And COVID-19' (ACNUDH y UA, 2020) <https://www.ohchr.org/Documents/Issues/Women/7ActionsFinal.pdf> acceso el 1 de febrero de 2021

75 Ibidem, p. 2.
} 
2. Acceso a la salud. Los derechos reproductivos y sexuales se han visto relegados y negados a las mujeres por parte de los gobiernos, excusándose en la necesidad de priorizar la crisis sanitaria. El informe en este caso ha abogado por incluir la perspectiva de género tanto en los aspectos relacionados con el acceso a la salud, como en aquellos ligados a las mujeres en riesgo por su posición en primera línea de emergencia sanitaria 76 .

3. Eliminación de la violencia de género. El peligroso aumento de la violencia de género, producto del confinamiento, no ha pasado inadvertido por ninguna de las organizaciones de derechos humanos. Las principales medidas se han orientado a elaborar una estructura reforzada de respuesta a casos de violencia de género, mejorando lo existente y robusteciendo las herramientas dadas al personal sanitario, judicial, policial... ${ }^{77}$

4. Acceso a comida, agua y sanidad. Aunque este aspecto no ha sido tratado con profundidad en el presente artículo, la pobreza y situación de vulnerabilidad también ha agudizado los casos de hambre y desnutrición, con especial hincapié en las mujeres refugiadas. Las herramientas que la ONU y la UA han propuesto un mayor y mejor acceso a la sanidad, al agua y a la comida; además de ampliar los programas de protección social para aquellas mujeres con mayores necesidades ${ }^{78}$.

5. Participación en la toma de decisiones. La presencia de las mujeres en primera línea de respuesta no se ha visto igualmente representada en los principales órganos de toma de decisiones, por lo que su inclusión en todos los aspectos de participación es sumamente necesaria para el logro de una pandemia con enfoque de género ${ }^{79}$.

6. Asistencia humanitaria. Los conflictos en la región del Norte de África y Medio Oriente han estado presentes desde antes de la pandemia y han llevado a las mujeres a una situación de especial vulnerabilidad dadas las condiciones de desplazamiento y refugio a las que se ven sometidas. En este sentido, el informe sugiere una mayor protección y asistencia humanitaria a estas mujeres y niñas ${ }^{80}$.

7. Colección de datos e información desagregados por género. Todas las problemáticas emanadas de las anteriores medidas podrían ser superadas si se dispusiese de un sistema de datos e información desagregado por género que pudiera registrar la evolución de la pandemia en las mujeres. Para ello, es imprescindible una recopilación de datos sobre cada uno de los

\footnotetext{
76 Ibidem, p. 3.

77 Ibidem, p. 4.

78 Ibidem, p. 5.

79 Ibidem, p. 6.

80 Ibidem, p. 7.
} 
ámbitos aquí estudiados y que sean susceptibles de llevar a las mujeres a una situación de mayor vulnerabilidad ${ }^{81}$.

Atendiendo al título del presente epígrafe, la posible situación futura de las mujeres dependerá de si los gobiernos de la región del Norte de África y Medio Oriente son capaces de implementar las medidas que desde las diferentes Organizaciones Internacionales y Regionales se están exigiendo. Un enfoque con perspectiva de género es imprescindible para que las sociedades en su conjunto puedan superar las secuelas de la actual pandemia, ya que el impacto en las mujeres se refleja en la sociedad en general ${ }^{82}$.

Por último, y pese a que en este informe no esté reseñado, sería de extrema urgencia valorar y atender la realidad de todas y cada una de las poblaciones no solo de la región analizada, sino de la integridad del globo. El hecho de que un territorio como es el del Sáhara Occidental sea invisibilizado y no tenido en cuenta por su estatus de Territorio No Autónomo y no de Estado ${ }^{83}$ viola gravemente los derechos humanos de las personas en territorio ocupado y en campamentos de personas refugiadas. Los informes de las principales organizaciones de referencia no han tenido en cuenta en prácticamente ninguna ocasión el territorio del Sáhara Occidental y la tesitura de las mujeres saharauis, por lo que una de las medidas también debería proponer visibilizar a todas las poblaciones de la región, independientemente de su estatus. Si no hay una atención temprana (por supuesto, con enfoque de género), se estará contribuyendo a la expansión del virus, al aumento de contagios, de fallecimientos y de desigualdad de género.

\section{5.- CONCLUSIONES}

Tras el análisis de los principales focos de desigualdad de género en el Norte de África y Medio Oriente a raíz de la pandemia provocada por el COVID-19, se han podido extraer una serie de conclusiones.

En primer lugar, en el precedente observado con el brote de enfermedad del Ébola y aunque las consecuencias fueron probablemente diferentes a las que se puedan generar con el COVID19 , sí que se pueden emplear para extrapolar la situación de un caso

\footnotetext{
${ }^{81}$ Ibidem, p. 8.

82 Como se advertía en el segundo epígrafe del presente artículo titulado "La situación de las mujeres en el Norte de África y Medio Oriente antes de la pandemia del COVID-19", la desigualdad de género en el salario no solo afecta a la mujer, sino que también supone la pérdida de capital humano para toda la región.

83 En ninguno de los informes reseñados se hace alusión al Sáhara Occidental como parte de la región del Norte de África y Medio Oriente. Para encontrar datos sobre el escenario actual del territorio se deben buscar noticias o informes especializados, en los cuales no se aborda el impacto hacia las mujeres por la falta de datos en general y desagregados por género.
} 
a otro, en concreto el impacto que las otras pandemias tuvieron en las mujeres.

En este sentido, se debería estudiar cómo los errores cometidos en otras pandemias pasadas se podrían volver a repetir si no se atendiera al género de una manera independiente. Buscando los factores que aumentan la vulnerabilidad y desigualdad de género se podrían dar soluciones por medio de políticas y medidas llevadas a cabo por los gobiernos para remediar lo que anteriormente no se realizó.

En segundo lugar, y directamente ligado a lo anterior, es necesario revertir la situación de invisibilización de la mujer en la pandemia. Su alta representación en primera línea de respuesta al COVID-19 contrasta con su baja presencia en la toma de decisiones y liderazgo. Este hecho está exacerbando aún más la brecha de género, al excluirla de los principales procesos en la evolución de la crisis sanitaria y de emergencia. Es por ello por lo que se torna indispensable una mayor participación de la mujer en todas las fases de evolución y de respuesta de los gobiernos. De no incitar a un cambio, se estarán afianzando aún más las normas de género tradicionales, relegando a las mujeres a un segundo plano y condenándolas a sufrir las mismas o peores secuelas en el futuro.

En tercer lugar, todo lo anteriormente expuesto no tendrá efecto si no se dispone de un sistema de datos desagregados por género que ponga de manifiesto los problemas estructurales ligados a las mujeres, para de esa manera poder ir a la base de los mismos y conseguir mitigar los efectos de la pandemia. Si no hay un conocimiento del problema no se puede dar una solución acertada.

En cuarto lugar, es imprescindible trasladar a la sociedad que aún es posible revertir el escenario actual. La pandemia del COVID19 podría representar una coyuntura para transformar las normas de género predominantes y tradicionalmente impuestas en la región. Existe aún la oportunidad de darle más protagonismo a la mujer y conseguir cumplir con el objetivo 5 de los ODS, destinado a lograr la igualdad de género y el empoderamiento de la mujer. Sin embargo, si no hay una pretensión de cumplir las medidas e introducir acciones, esta pandemia no solo dejará a las mujeres del Norte de África y Medio Oriente ( $y$ de todo el mundo) en el punto de partida, sino que además constituirá un retroceso en el logro de un rol y una mayor participación de la mujer en los procesos de toma de decisiones.

Como reflexión final, una pandemia puede acrecentar los problemas existentes en los derechos humanos de las distintas sociedades, pero no tiene la capacidad de discriminar. En cambio, las decisiones, medidas y respuestas dadas por los gobiernos y los órganos de toma de decisiones de los países sí. Por consiguiente, son ellos los que deben proporcionar las herramientas de cambio.

Tal y como se pronunciaba una organización feminista de Líbano: La pandemia ha magnificado todos los problemas $y$ violaciones de derechos humanos, incluidos los derechos humanos 
básicos a la educación, la salud, la alimentación y la ropa. (...) Estos problemas flotaron a la superficie debido a la falta de estructuras o mecanismos de respuesta ${ }^{84}$.

\section{6.- BIBLIOGRAFÍA}

ACNUDH y UA, '7 Possible Actions- Women'S Rights And COVID-19' (ACNUDH Y UA, 2020) <https://www.ohchr.org/Documents/Issues/Women/7ActionsFi nal.pdf> acceso el 1 de febrero de 2021

Amnistía Internacional, 'Los Derechos Humanos En Oriente Medio Y El Norte De África' (Amnistía Internacional, 2020) <https://www.amnesty.org/download/Documents/MDE0113572 020SPANISH.pdf> acceso el 1 de febrero de 2021

Carboni J, and Gimeno Martín J, 'Inmóviles, Pero No Quietos. La Sedentarización De Los Saharauis Como Estrategia De Adaptación Y Respuesta A La Supervivencia. Sobre La Posibilidad De Un Nomadismo Inmóvil' [2021] Tabula Rasa

CARE, 'One More Setback For Refugees In MENA -Especially Women And Girls' (CARE International, 2020) $<$ https://reliefweb.int/sites/reliefweb.int/files/resources/CARE_ MENA_Rapid_Gender_Analysis_Summary.pdf> acceso el 1 de febrero de 2021

CARE, 'Rapid Gender Analysis - COVID-19. Middle East And North Africa Region' (CARE International, 2020) <https://reliefweb.int/sites/reliefweb.int/files/resources/CAREMENA-COVID-19-RGA-200720201.pdf> acceso el 1 de febrero de 2021

Caruso F, 'COVID-19 And Conflict Mediation: Women, Not The Pandemic Can Revive Diplomacy' [2020] Istituto Affari Internazionali, 52

Díaz C, y Galanzini N, 'Una Pandemia En La Sombra De La COVID-10' [2020] Fundación Mujeres por África

Eghtessadi $R$ y otros, 'Safeguarding Gains In The Sexual And Reproductive Health And AIDS Response Amidst COVID-19: The Role Of African Civil Society' (2020) 100 International Journal of Infectious Diseases

Fernández-Luis S y otras, '¿Qué Sabemos Del Impacto De Género En La Pandemia De La COVID-19?' [2020] ISGlobal, Serie Covid-19 y estrategia de respuesta

IASC, 'Humanitarian Crisis In West Africa (Ebola). Gender Alert' (Inter-Agency Standing Committee (IASC, 2014) <https://www.globalhealth.org/wp-content/uploads/IASC-

84 WILPF, 'COVID-19 And Gender Justice: Feminists In MENA Defying Global Structural Failure' (Women's International League for Peace and Freedom (WILPF), 2021) <https://www.wilpf.org/wp-content/uploads/2020/10/WILPF_COVID-19MENA-Consultation_Web.pdf > acceso el 1 de febrero de 2021 p. 12. 
Gender-Reference-Group-Gender-Alert-WEST-AFRICA-EBOLA19-Sept-2014.pdf> acceso el 1 de febrero de 2021

Interagency Network on Women and Gender Equality (IANWGE), 'IANWGE Compendium On Integrating Gender Considerations In The Response To COVID-19: Key Messages And Actions From Entities' (Interagency Network on Women and Gender Equality (IANWGE), 2020) <https://www.unwomen.org//media/headquarters/attachments/sections/library/publications/ 2020/ianwge-compendium-on-integrating-genderconsiderations-in-the-response-to-covid-19en.pdf?la=en\&vs $=5806>$ acceso el 1 de febrero de 2021

Meagher K, Singh N, y Patel P, 'The Role Of Gender Inclusive Leadership During The COVID-19 Pandemic To Support Vulnerable Populations In Conflict Settings' (2020) 5 BMJ Global Health

OCDE, 'COVID-19 Crisis In The MENA Region: Impact On Gender Equality And Policy Responses' (Organización para la Cooperación y el Desarrollo Económicos (OCDE), 2020) $<$ https://read.oecd-ilibrary.org/view/?ref=134_134470w95kmv8khl\&title=COVID-19-crisis-in-the-MENA-regionimpact-on-gender-equality-and-policy-responses> acceso el 1 de febrero de 2021

OCDE, 'COVID-19 crisis response in MENA countries' (Organización para la Cooperación y el Desarrollo Económicos (OCDE), 2020) $<$ https://read.oecd-ilibrary.org/view/?ref=129_1299194li7bq8asv\&title=COVID-19-Crisis-Response-in-MENACountries > acceso el 1 de febrero de 2021

ONU Mujeres, 'COVID-19 Crisis Update And UN Women Response Morocco' (ONU Mujeres, 2020) <https://www2.unwomen.org//media/field\%20office\%20arab\%20states/attachments/publicat ions/2020/04/covid $19 \% 20$ crisis\%20update\%20and\%20un\%20 women\%20response160420.pdf?la=en\&vs $=5039>$ acceso el 1 de febrero de 2021

ONU Mujeres, 'COVID-19: Gendered Impacts of the Pandemic in Palestine and Implications for Policy and Programming Findings of a Rapid Gender Analysis of COVID-19 in Palestine' (ONU Mujeres, 2020) <https://www.un.org/unispal/wpcontent/uploads/2020/06/UNWOMENRPT_090620.pdf> acceso el 1 de febrero de 2021

ONU Mujeres, 'COVID-19 y conflictos: Fomentar la participación sustantiva de las mujeres en los procesos de paz y alto el fuego. Documento de políticas no. 19' (ONU Mujeres, 2020) < https://www.unwomen.org/-

/media/headquarters/attachments/sections/library/publications/ 2020/policy-brief-covid-19-and-conflict-

es.pdf?la=es\&vs=3825> acceso el 1 de febrero de 2021

ONU Mujeres, 'Press Release: Women'S Organizations In The Arab States Region Join UN Secretary-General António Guterres'S 
Call For Ceasefire In The Face Of COVID-19' (ONU Mujeres, 2020)

<https://www.unwomen.org/en/news/stories/2020/5/press-

release-women-organizations-in-the-arab-states-call-for-

ceasefire-in-the-face-of-covid19> acceso el 1 de febrero de 2021

ONU Mujeres, 'The impact of COVID-19 on Gender Equality in the Arab Region' (ONU Mujeres, 2020) <https://www2.unwomen.org//media/field\%20office\%20arab\%20states/attachments/publicat ions/2020/04/impact\%20of\%20covid\%20on\%20gender\%20eq uality\%20-\%20policy\%20brief.pdf?la=en\&vs=4414> acceso el 1 de febrero de 2021

ONU Mujeres, 'Women's needs and gender equality in Egypt's COVID19 response' (ONU Mujeres, 2020) <https://www2.unwomen.org/-

/media/field\%20office\%20arab\%20states/attachments/publicat ions/2020/04/unwomen_brief_covid-

19_egypt_response.pdf?la=en\&vs $=3919>$ acceso el 1 de febrero de 2021

ONU Mujeres, 'Women's needs and gender equality in Lebanon's COVID-19 response' (ONU Mujeres, 2020) $<$ https://www2.unwomen.org/-

/media/field\%20office\%20arab\%20states/attachments/publicat ions/2020/03/updated\%20lebanon\%20brief/gender\%20and\%2 0covid_english.pdf?la=en\&vs $=403>$ acceso el 1 de febrero de 2021

ONU SIDA, 'New Awareness Campaign On Gender-Based Violence In The Middle East And North Africa' (ONU SIDA, 2020) <https://www.unaids.org/en/resources/presscentre/featurestori es/2020/november/20201125_awareness-campaign-gender-

based-violence-middle-east-north-africa> acceso el 1 de febrero de 2021

ONU, 'Policy Brief: The Impact Of COVID-19 On Women' (ONU, 2020) <https://www.un.org/sites/un2.un.org/files/policy_brief_on_co vid_impact_on_women_9_april_2020.pdf> acceso el 1 de febrero de 2021

ONU, 'Policy Note on the United Nations Secretary-General's Call for a Global Ceasefire: Challenges and Opportunities' (Mediation Support Unit, Policy \& Mediation Division, 2020) https://peacemaker.un.org/sites/peacemaker.un.org/files/Polic y\%20Note\%20Ceasefire\%20Call\%20Challenges\%200pportuniti es_0_0.pdf> acceso el 1 de febrero de 2021

Pearce E, 'Disability Considerations In GBV Programming During The COVID-19 Pandemic' [2020] GBV AoR Help Desk

Peterman A y otras, 'Pandemics And Violence Against Women And Children' [2020] Center for Global Development. Working Paper 528 
Ravindran S, and Shah M, 'Unintended Consequences Of Lockdowns: COVID-19 And The Shadow Pandemic' [2020] National Bureau of Economic Research Working Paper Series

Rivera C y otras, 'Gender Inequality And The COVID-19 Crisis: A Human Development Perspective' (Programa de las Naciones Unidas para el Desarrollo (PNUD, 2020) $<$ http://hdr.undp.org/sites/default/files/covid-

19_and_human_development_-_gender_dashboards_final.pdf> acceso el 1 de febrero de 2021

Smith J, 'Overcoming The 'Tyranny Of The Urgent': Integrating Gender Into Disease Outbreak Preparedness And Response' (2019) 27 Gender \& Development

Solanas Cardín M, 'La Crisis Del COVID-19 Y Sus Impactos En La Igualdad De Género' [2020] Real Instituto Elcano

UNFPA, ' Daring to Ask, Listen, and Act: A Snapshot of the Impacts of COVID-19 on Women and Girls' rights and sexual and reproductive health' (United Nations Population Fund (UNFPA), 2020) https://reliefweb.int/sites/reliefweb.int/files/resources/2020051 1_Daring\%20to\%20ask\%20Rapid\%20Assessment\%20Report_F INAL.pdf > acceso el 1 de febrero de 2021

UNFPA, 'Arab States Region COVID-19. Situation Report No. 1' (United Nations Population Fund (UNFPA), 2020) <https://www.unfpa.org/sites/default/files/resourcepdf/ASRO_COVID-19_UNFPA_Sitrep_No.1.pdf> acceso el 1 de febrero de 2021

Williams T, y Pontalti K, 'Responding To The Shadow Pandemic: Taking Stock Of Gender-Based Violence Risks And Responses During COVID-19. Child Protection Learning Brief' (UNICEF, 2021) <https://www.unicef.org/media/76916/file/GenderBased-Violence-in-Emergencies-CP-Learning-Brief-Aug2020.pdf> acceso el 1 de febrero de 2021

WILPF, 'COVID-19 And Gender Justice: Feminists In MENA Defying Global Structural Failure' (Women's International League for Peace and Freedom (WILPF), 2021) <https://www.wilpf.org/wpcontent/uploads/2020/10/WILPF_COVID-19-MENAConsultation_Web.pdf $>$ acceso el 1 de febrero de 2021

Wodon Q, y de la Brière B, 'Unrealized Potential: The High Cost Of Gender Inequality In Earnings' (Banco Mundial, 2018) <https://openknowledge.worldbank.org/bitstream/handle/1098 6/29865/126579-Public-on-5-30-18-WorldBank-

GenderInequality-Brief-v13.pdf?sequence $=1$ \&isAllowed $=y>$ acceso el 1 de febrero de 2021 